INJE-TP-99-9, hep-th/9912288

\title{
S-wave absorption of scalars by noncommutative D3-branes
}

\author{
Y.S. Myung, Gungwon Kang, and H.W. Lee \\ Department of Physics, Inje University, Kimhae 621-749, Korea
}

\begin{abstract}
On the supergravity side, we study the propagation of the RR scalar and the dilaton in the D3-branes with NS $B$-field. To obtain the noncommutative effect, we consider the case of $B \rightarrow \infty(\theta \rightarrow \pi / 2)$. We approximate this as the smeared D1-brane background with $F_{5}=H=0$. In this background, the RR scalar induces an instability of the near-horizon geometry. However, it turns out that the RR scalar is nonpropagating, while the dilaton is a physically propagating mode. We calculate the s-wave absorption cross section of the dilaton. One finds $\left.\sigma_{0}^{\phi}\right|_{B \rightarrow \infty} \sim\left(\tilde{\omega} \tilde{R}_{\frac{\pi}{2}}\right)^{8.9} / \omega^{5}$ in the leading-order while $\left.\sigma_{0}^{\phi}\right|_{B=0} \sim\left(\tilde{\omega} R_{0}\right)^{8} / \omega^{5}$ in the D3-branes without $B$-field. This means that although the dilaton belongs to a minimally coupled scalar in the absence of $B$-field, it becomes a sort of fixed scalar in the limit of $B \rightarrow \infty$.
\end{abstract}




\section{INTRODUCTION}

Recently noncommutative geometry has attracted much interest in studying on string and M-theory in the $B$-field [1] [7]. For simplicity, we consider supergravity solutions which are related to D3-branes with NS B field. According to the AdS/CFT correspondence [8], the near horizon geometry of $\mathrm{D}=7$ black hole solution can describe the large $N$ limit of noncommutative super Yang-Mills theory (NCSYM). We take a decoupling limit to isolate the near horizon geometry from the remaining one. It turns out that the noncommutativity affects the ultra violet(UV) regime, leaving the infra red(IR) regime of the Yang-Mills dynamics unchanged. The NCSYM is thus not useful for studying the theory at short distances. It is well known that an NCSYM with the noncommutativity scale $\Delta$ on a torus of size $\Sigma$ is equivalent to an ordinary supersymmetric Yang-Mills theory (OSYM) with a magnetic flux provided that $\Theta=\Delta^{2} / \Sigma^{2}$ is a rational number [9]. The equivalence between the NCSYM and the OSYM can be understood from the T-duality of the corresponding string theory. Hence the OSYM with $B$-field is the proper description in the UV region, while the NCSYM takes over in the IR region. Actually, the noncommutativity comes from the $B \rightarrow \infty$ limit of the ordinary theories [5, 6, 10].

We remind the reader that, aside the entropy, there exists an important dynamical quantity, "the greybody factor(absorption cross section)" for the quantum black hole [12 [15]. On the string side, there was a calculation for the absorption of scalars into the noncommutative D3-branes [16]. However, the authors have not considered the RR sectors in Ref. [16].

Myung, Kang, and Lee [17]have studied the quantum aspect of the D3-brane black hole in $B_{23}$-field background using a minimally coupled scalar. Such minimally coupled field might describe fluctuations of the off-diagonal gravitons polarized parallel to the brane $\left(h_{a b}, a, b=0,1,2,3\right)$. They derived the exact form of the absorption $\operatorname{cross} \operatorname{section}\left(\sigma_{l}\right)$ in $B$-field on the supergravity side. It is well known that the cross section can be extracted from the solution to the linearized equation after the diagonalization. It turns out that

$\sigma_{l}^{B \neq 0}>\sigma_{l}^{B=0}$. This implies that the presence of the $B$-field suppresses the curvature effect 
surrounding the $\mathrm{D}=7$ black hole. As a result, it comes out the increase of greybody factor.

Recently, Kaya [18 has calculated the absorption cross section for the RR scalar in the D3-brane with the large $B$-field. He claimed that the greybody factor does not change even if the $B$-field is large. Hence the RR scalar turns out to be a minimally coupled scalar with $B=0$ even for the presence of the large $B$-field.

In this paper we study the propagations of the RR $\operatorname{scalar}(\chi)$ and the dilaton $(\Phi)$ by D3branes with $B$-field along their world volume directions $\left(x_{2}, x_{3}\right)$. Especially we are interested in the case of $B \rightarrow \infty$. Here we use all information contained in the equations of motion, the Bianchi identities, and the gauge condition for graviton. In the absence of $B$-field, such fields as well as gravitons polarized parallel to the D3-brane belong to minimally coupled scalars. However, in the presence of $B$-field, these scalars are coupled to the background nonminimally. In this sense, we may regard such fields as the fixed scalars. Actually, in the smeared D1-brane background, the dilaton(RR scalar) turns out to be (non)propagating modes. It is very important to test whether or not there is a change in the absorption cross sections of the dilaton between $B=0$ and $B \rightarrow \infty$ cases.

The organization of this paper is as follows. In Sec.T, we briefly review the field equations relevant for our study. Here we study $B \rightarrow \infty$ limit carefully and introduce the smeared D1-brane black hole. Sec III is devoted to analyzing the perturbations around the smeared D1-brane background. The propagation of the RR scalar is investigated in Sec.[V]. This induces an instability of the near-horizon geometry. Sec. $\square$ deals with the propagation of the dilaton with the dilaton gauge. In Sec.V1, we study the propagation of the dilaton with the harmonic gauge and obtain its absorption cross section. We discuss our results in Sec.VII. Finally we present the smeared D1-brane solution in Appendix A.

\section{FORMALISM}

We start with the low energy limit of type IIB superstring action in the Einstein $\operatorname{frame}\left(g_{M N}=e^{-\Phi / 2} G_{M N}\right)$ [12] 


$$
\begin{aligned}
S_{10}^{E}= & \frac{1}{2 \kappa_{10}^{2}} \int d^{10} x\left[\sqrt { - g } \left\{R-\frac{1}{2}(\nabla \Phi)^{2}-\frac{1}{12} e^{-\Phi}\left(\partial B_{2}\right)^{2}-\frac{1}{2} e^{2 \Phi}(\partial \chi)^{2}\right.\right. \\
& \left.\left.-\frac{1}{12} e^{\Phi}\left(\partial C_{2}-\chi \partial B_{2}\right)^{2}-\frac{1}{4 \cdot 5 !} F_{5}^{2}\right\}-\frac{1}{2 \cdot 4 ! \cdot(3 !)^{2}} \epsilon_{10} C_{4} \partial C_{2} \partial B_{2}\right]
\end{aligned}
$$

where $\Phi$ is the dilaton, $\chi$ the RR scalar, $B_{2}$ the NS two form, $C_{2}$ the RR two form, and $C_{4}$ the RR four form. And one has

$$
\begin{aligned}
& H_{M N P}=\left(\partial B_{2}\right)_{M N P}=3 \partial_{[M} B_{N P]}, \quad F_{3 M N P}=\left(\partial C_{2}\right)_{M N P}=3 \partial_{[M} C_{2 N P]}, \\
& \left(\partial C_{4}\right)_{M N P Q R}=5 \partial_{[M} C_{4 N P Q R]}, \quad F_{5}=\partial C_{4}+5\left(B_{2} \partial C_{2}-C_{2} \partial B_{2}\right) .
\end{aligned}
$$

with the self-duality constraint $F_{5}=\tilde{F}_{5}$ at the level of the equations of motion. The relevant equations of motion lead to [19]

$$
\begin{aligned}
& \nabla^{2} \chi+2 \nabla \Phi \nabla \chi+\frac{e^{-\Phi}}{6}\left(F_{3}-\chi H\right) \cdot H=0 \\
& \nabla^{2} \Phi+\frac{1}{12}\left\{e^{-\Phi} H^{2}-e^{\Phi}\left(F_{3}-\chi H\right)^{2}\right\}-e^{2 \Phi}(\nabla \chi)^{2}=0 \\
& \nabla_{M}\left(e^{-\Phi} H^{M P Q}\right)-\nabla_{M}\left\{\chi e^{\Phi}\left(F_{3}-\chi H\right)^{M P Q}\right\}+\frac{2}{3} F^{P Q R S T} F_{3 R S T}=0, \\
& \nabla_{M}\left\{e^{\Phi}\left(F_{3}-\chi H\right)^{M P Q}\right\}-\frac{2}{3} F^{P Q R S T} H_{R S T}=0, \\
& \nabla_{M} F^{M P Q R S}=0 .
\end{aligned}
$$

In the string frame, Eqs. (3)-(7) take the following forms

$$
\begin{aligned}
& \nabla_{s}^{2} \chi+\frac{1}{6}\left(F_{3 s}-\chi H_{s}\right) \cdot H_{s}=0 \\
& \nabla_{s}^{2} \Phi-2\left(\nabla_{s} \Phi\right)^{2}+\frac{1}{12}\left\{H_{s}^{2}-e^{2 \Phi}\left(F_{3 s}-\chi H_{s}\right)^{2}\right\}-e^{2 \Phi}\left(\nabla_{s} \chi\right)^{2}=0 \\
& \nabla_{s M}\left(e^{-2 \Phi} H_{s}^{M P Q}\right)-\nabla_{s M}\left\{\chi\left(F_{3 s}-\chi H_{s}\right)^{M P Q}\right\}+\frac{2}{3} F_{s}^{P Q R S T} F_{3 s R S T}=0 \\
& \nabla_{s M}\left(F_{3 s}-\chi H_{s}\right)^{M P Q}-\frac{2}{3} F_{s}^{P Q R S T} H_{s R S T}=0 \\
& \nabla_{s M} F_{s}^{M P Q R S}=0 .
\end{aligned}
$$

In addition, we need the remaining Maxwell equations, as three Bianchi identities

$$
\partial_{[M} H_{N P Q]}=\partial_{[M} F_{N P Q]}=\partial_{[M} F_{N P Q R S]}=0
$$

The solution of $\mathrm{D}=7$ extremal black hole for the D3-branes with non-zero $B_{23}$-field is given as follows in the $\mathrm{D}=10$ string frame [5] 


$$
\begin{aligned}
& d s_{s}^{2}=f^{-\frac{1}{2}}\left\{-d x_{0}^{2}+d x_{1}^{2}+h\left(d x_{2}^{2}+d x_{3}^{2}\right)\right\}+f^{\frac{1}{2}}\left(d r^{2}+r^{2} d \Omega_{5}^{2}\right), \\
& f=1+\frac{R_{\theta}^{4}}{r^{4}}, \quad h^{-1}=\sin ^{2} \theta f^{-1}+\cos ^{2} \theta \\
& \bar{B}_{s 23}=\tan \theta f^{-1} h, \quad e^{2 \bar{\Phi}}=g^{2} h, \\
& \bar{F}_{s 01 r}=\frac{1}{g} \sin \theta \partial_{r}\left(f^{-1}\right), \quad \bar{F}_{s 0123 r}=\frac{1}{g} \cos \theta h \partial_{r}\left(f^{-1}\right) .
\end{aligned}
$$

From now on we work in the string frame and thus neglect the subscript "s". Here the asymptotic value of $B$-field is $\bar{B}_{23}^{\infty}=\tan \theta$ and the parameter $R_{\theta}$ is defined by $\cos \theta R_{\theta}^{4}=R_{0}^{4}(=$ $\left.4 \pi g N \alpha^{2}\right) . \quad N$ is the number of the D3-branes and $g=g_{\infty}$ is the string coupling constant. It is obvious that for $\theta=0(h=1)$ one recovers the ordinary D3-brane black hole with the standard $\mathrm{AdS}_{5} \times \mathrm{S}^{5}$ geometry in the near horizon. In this case we have $\bar{F}_{0123 r}=\frac{1}{g} \partial_{r}\left(f^{-1}\right)$, its dual $\left(\tilde{\bar{F}}_{5}\right)$, and $e^{2 \bar{\Phi}}=g^{2}$.

For $\theta \rightarrow \pi / 2(h \rightarrow f)$, however, one finds the D3-brane black hole in the very large $B$-field and thus the effect of noncommutativity appears. Here one finds a deviation from $\mathrm{AdS}_{5} \times \mathrm{S}^{5}$ in the near horizon. However, it is known that in order to make connection to noncommutative geometry, $\theta \rightarrow \frac{\pi}{2}(B \rightarrow \infty)$ limit must be carefully taken. In addition, we need a double scaling limit of $g N \rightarrow \infty, \omega^{4} \alpha^{\prime 2} \rightarrow 0$ to keep the expansion parameter $\omega^{4} R_{0}^{4}$ very small in the calculation of the absorption cross section. This implies the decoupling limit of $g \rightarrow 0, \alpha^{\prime} \rightarrow 0: g N \gg 1$ and the low-energy limit $(\omega \rightarrow 0)$. Here we wish to take into account all of these limits only by taking $\alpha^{\prime} \rightarrow 0$ :

$$
\tan \theta=\frac{\tilde{b}}{\alpha^{\prime}}, \quad g=\alpha^{\prime} \tilde{g}, \quad N=\frac{\tilde{N}}{\alpha^{\prime 2}},
$$

where $\tilde{b}, \tilde{g}, \tilde{N}$ stay fixed [5]. Further this implies that

$$
\lim _{\theta \rightarrow \frac{\pi}{2}}\left(R_{\theta}^{4}=\frac{R_{0}^{4}}{\cos \theta}\right)=4 \pi \tilde{g} \tilde{b} \tilde{N} \equiv R_{\frac{\pi}{2}}^{4} .
$$

This means that $\lim _{\theta \rightarrow \frac{\pi}{2}} R_{\theta}^{4}$ is nearly independent of $\theta$ and is finite with $R_{\frac{\pi}{2}}^{4} \gg R_{0}^{4}$. But we must choose the low-energy $\operatorname{limit}(\omega \rightarrow 0)$ to keep $\omega^{4} R_{\frac{\pi}{2}}^{4}$ small. Under this condition, one finds

$$
\bar{H}_{r 23}=\tan \theta \partial_{r}\left(f^{-1} h\right) \underset{\alpha^{\prime} \rightarrow 0}{\stackrel{\theta \rightarrow \pi / 2}{\rightarrow}} \alpha^{\prime} \rightarrow 0
$$




$$
\begin{aligned}
\bar{F}_{01 r} & =\frac{1}{g} \sin \theta \partial_{r}\left(f^{-1}\right) \underset{\alpha^{\prime} \rightarrow 0}{\stackrel{\theta \rightarrow \pi / 2}{\longrightarrow}} \frac{1}{\alpha^{\prime}} \rightarrow \infty, \\
\bar{F}_{0123 r} & =\frac{1}{g} \cos \theta \partial_{r}\left(f^{-1}\right) \underset{\alpha^{\prime} \rightarrow 0}{\stackrel{\theta \rightarrow \pi / 2}{\longrightarrow}} \longrightarrow \text { finite. }
\end{aligned}
$$

Here one obtains a sequence of $\bar{F}_{01 r} \gg \bar{F}_{0123 r} \gg \bar{H}_{r 23}$ in this limit. As a result, although the flux of the RR five-form $\left(\bar{F}_{5}\right)$ counts the rank of the noncommutative gauge group, this is very small in comparison with the $\mathrm{RR}$ three-form $\left(\bar{F}_{3}\right)$ in the limit of $\theta \rightarrow \frac{\pi}{2}(B \rightarrow \infty)$. Hence we can neglect the effect of $\bar{F}_{5}$ and $\bar{H}$ on the absorption cross section in favor of $\bar{F}_{3}$.

In the case of $\bar{F}_{5}=\bar{H}=0, e^{2 \bar{\Phi}}=g^{2} f, \bar{F}_{01 r}=\frac{1}{g} \partial_{r}\left(f^{-1}\right)$, one obtains the smeared D1brane solution in Appendix A. We may regard this solution as the simple one to include the noncommutative effect through $\tilde{R}_{\frac{\pi}{2}}^{4} \gg R_{0}^{4}$ in $\tilde{f}=1+\tilde{R}_{\frac{\pi}{2}}^{4} / r^{4}$. Here $\tilde{R}_{\frac{\pi}{2}}^{4}=(1+\epsilon) R_{\frac{\pi}{2}}^{4}$ with $\epsilon=k^{2} / \tilde{\omega}^{2}=k^{2} / \omega^{2}\left(1-k^{2} / \omega^{2}\right)^{-1}$.

\section{PERTURBATIONS}

Now let us introduce the perturbations to derive the greybody factor as 20

$$
\begin{aligned}
G_{M N} & =\bar{G}_{M N}+h_{M N} \\
\chi & =0+\eta, \\
\Phi & =\bar{\Phi}+\phi \\
F_{01 r} & =\bar{F}_{01 r}+f_{01 r}=\bar{F}_{01 r}\left(1+f_{3}\right), \\
H_{r 23} & =\bar{H}_{r 23}+h_{r 23}=\bar{H}_{r 23}\left(1+h_{3}\right), \\
F_{0123 r} & =\bar{F}_{0123 r}+f_{0123 r}=\bar{F}_{0123 r}\left(1+f_{5}\right)
\end{aligned}
$$

with all other perturbations to be zero. For the perturbations above, we keep the background symmetry up to the linearized level. General fluctuations give a complicated system of differential equations:

$$
\begin{aligned}
& \nabla^{2} \eta-\frac{1}{6} \bar{H}^{2} \eta=0 \\
& -h^{M N} \nabla_{M} \nabla_{N} \bar{\Phi}-\bar{G}^{M N} \delta \Gamma_{M N}^{P} \nabla_{P} \bar{\Phi}+\nabla^{2} \phi-4 \nabla \bar{\Phi} \cdot \nabla \phi
\end{aligned}
$$




$$
\begin{aligned}
&+ 2 \nabla_{M} \bar{\Phi} \nabla_{N} \bar{\Phi} h^{M N}+\frac{1}{12}\left(2 \bar{H}^{2} h_{3}-3 \bar{H}_{M N Q} \bar{H}^{P N Q} h_{P}^{M}\right) \\
&-\frac{e^{2 \bar{\Phi}}}{12}\left\{2 \bar{F}_{3}^{2}\left(\phi+f_{3}\right)-3 \bar{F}_{M N Q} \bar{F}^{P N Q} h_{P}^{M}\right\}=0, \\
& e^{-2 \bar{\Phi}}\left\{\left(\nabla_{M}-2 \nabla_{M} \bar{\Phi}\right)\left(\bar{H}^{M N P} h_{3}\right)-\left(\nabla_{M} h_{Q}^{N}\right) \bar{H}^{M Q P}+\left(\nabla_{M} h_{Q}^{P}\right) \bar{H}^{M Q N}\right. \\
&\left.-\left(\nabla_{M} \hat{h}_{Q}^{M}\right) \bar{H}^{Q N P}-h_{Q}^{M} \nabla_{M} \bar{H}^{Q N P}-2\left(\nabla_{M} \phi\right) \bar{H}^{M N P}\right\} \\
&-2 \nabla_{M}\left(e^{-2 \bar{\Phi}} \bar{H}^{M N P}\right) \phi-\nabla_{M}\left(\bar{F}^{M P Q} \eta\right)+\frac{2}{3} \bar{F}^{P Q R S T} \bar{F}_{R S T}\left(f_{3}+f_{5}\right)=0, \\
& \nabla_{M}\left(\bar{F}^{M N P} f_{3}\right)-\left(\nabla_{M} h_{Q}^{N}\right) \bar{F}^{M Q P}+\left(\nabla_{M} h_{Q}^{P}\right) \bar{F}^{M Q N}-h_{Q}^{M} \nabla_{M} \bar{F}^{Q N P} \\
& \quad-\left(\nabla_{M} \hat{h}_{Q}^{M}\right) \bar{F}^{Q N P}-\nabla_{M}\left(\bar{H}^{M P Q} \eta\right)-\frac{2}{3} \bar{F}^{P Q R S T} \bar{H}_{R S T}\left(h_{3}+f_{5}\right)=0, \\
& \nabla_{M}\left(\bar{F}^{M N P Q R} f_{5}\right)-4 \bar{F}^{M T[P Q R} \nabla_{M} h_{T}^{N]}-\left(\nabla_{M} \hat{h}_{T}^{M}\right) \bar{F}^{T N P Q R}-h_{T}^{M} \nabla_{M} \bar{F}^{T N P Q R}=0
\end{aligned}
$$

with $\delta \Gamma_{M N}^{P}=\frac{1}{2} \bar{G}^{P Q}\left(\nabla_{M} h_{N Q}+\nabla_{N} h_{M Q}-\nabla_{Q} h_{M N}\right)$. Here we have a relation of $\bar{G}^{M N} \delta \Gamma_{M N}^{P}=$ $\nabla_{M} \hat{h}^{M P}, \hat{h}^{M P}=h^{M P}-\frac{1}{2} \bar{G}^{M P} h$ with $h=h_{T}^{T}$. Let us check the order of $g$ in each equation. To obtain all consistent linearized equations, we have to scale $\eta$ in Eqs.(26), (28) and (29) as $\eta / g$. Furthermore we find from three Bianchi identities in Eq.(13) with Eqs.(23)-(25) that

$$
\begin{aligned}
f_{3}, f_{5} & \rightarrow \text { propagating modes, } \\
h_{3} & \rightarrow \text { nonpropagating mode. }
\end{aligned}
$$

This means that the NS $B$-field is considered as a tool for giving the noncommutative effect but it does not belong to the physically propagating field. For the graviton modes, we may use either the dilaton gauge [21]

$$
\nabla_{M} \hat{h}^{M P}=h^{M N} \Gamma_{M N}^{P}
$$

or the harmonic gauge 22

$$
\nabla_{M} \hat{h}^{M P}=0
$$

Although a choice of gauge condition does not eliminate all of the gauge freedom, it simplifies the perturbation equations. We note here that, although the equation (26) is a decoupled one for the RR scalar $(\eta)$, Eqs.(28) and (29) contain information for $\eta$. Kaya considered 
Eq.(26) only in ref. [18]. As can be seen the dilaton equation takes a very complicate form coupled with various other fields. To decouple $\phi$ from the remaining fields, we have to do some further work. Hence we separate the RR scalar from the dilaton. Let us first investigate the RR scalar.

\section{RR SCALAR PROPAGATION}

Because of the RR scalar equation (26) is completely decoupled from others, we start with an arbitrary $B(\theta)$. A way to obtain the noncommutative effect is to include the momentum dependence along the world volume directions [5]. This is because the $B_{23}$-field is set up along these directions. Hence $x_{2}, x_{3}$ become noncommuting coordinates. Now let us consider the spacetime dependence

$$
\eta\left(t, x_{1}, x_{2}, x_{3}, r, \theta_{i}\right)=e^{-i \omega t} e^{i\left(k_{1} x_{1}+k_{2} x_{2}+k_{3} x_{3}\right)} Y_{l}\left(\theta_{1}, \theta_{2}, \cdots, \theta_{5}\right) \eta^{l}(r)
$$

with $\bar{\nabla}_{\theta_{i}}^{2} Y_{l}\left(\theta_{i}\right)=-l(l+4) Y_{l}\left(\theta_{i}\right) . Y_{l}\left(\theta_{i}\right)$ denotes spherical harmonics on $S^{5}$ with unit radius. Here $\eta^{l}(r)$ is the radial part of the $l$-partial wave of energy $\omega$. Then Eq. (26) takes the form

$$
\left\{\frac{\partial^{2}}{\partial r^{2}}+\frac{5}{r} \frac{\partial}{\partial r}+\frac{h^{\prime}}{h} \frac{\partial}{\partial r}-\frac{l(l+4)}{r^{2}}+\left(\omega^{2}-k_{1}^{2}\right) f-\frac{\left(k_{2}^{2}+k_{3}^{2}\right) f}{h}-\frac{f^{\prime 2} \sin ^{2} \theta \cos ^{2} \theta h^{2}}{f^{3}}\right\} \eta^{l}=0
$$

with $f^{\prime}=\frac{d}{d r} f$. If $k_{1}=k_{2}=k_{3}=0$, this is exactly the equation that Kaya has considered in the first version of ref. [18].

If $\theta=0$ (B-field is turned off) and $l=0$, one finds that Eq.(34) reduces to the s-wave minimally coupled scalar $(\varphi)$ equation in the $\mathrm{D}=7$ black hole background [12]

$$
\left\{\frac{\partial^{2}}{\partial r^{2}}+\frac{5}{r} \frac{\partial}{\partial r}+\tilde{\omega}^{2}\left(1+\frac{R_{0}^{4}}{r^{4}}\right)\right\} \varphi^{0}=0
$$

with $\tilde{\omega}=\sqrt{\omega^{2}-k_{1}^{2}-k_{2}^{2}-k_{3}^{2}} \simeq \omega\left(1-\frac{k^{2}}{2 \omega^{2}}\right), k^{2}=k_{1}^{2}+k_{2}^{2}+k_{3}^{2}, \omega^{2}>k^{2}$. The s-wave absorption cross section for Eq. (35) can be obtained from the solution to Mathieu's equation as [13]

$$
\left.\sigma_{0}^{\varphi}\right|_{B=0}=\frac{\pi^{4}\left(\tilde{\omega} R_{0}\right)^{8}}{8 \omega^{5}}
$$


in the leading-order calculation. We note here that $\left.\sigma_{0}^{\eta}\right|_{B=0}=\left.\sigma_{0}^{\phi}\right|_{B=0}=\left.\sigma_{0}^{\varphi}\right|_{B=0}$, because fluctuations of both RR scalar and the dilaton fields belong to minimally coupled scalars when $B$-field is absent. For an arbitrary $B$, the corresponding equations for a minimally coupled field $\varphi$ is given by

$$
\left\{\frac{\partial^{2}}{\partial r^{2}}+\frac{5}{r} \frac{\partial}{\partial r}+\tilde{\omega}^{2}\left(1+\frac{\tilde{R}_{\theta}^{4}}{r^{4}}\right)\right\} \varphi_{B}^{0}=0
$$

where $\tilde{R}_{\theta}^{4}=(1+\epsilon) R_{\theta}^{4}$ with $\epsilon(\theta)=\frac{k^{2}}{\tilde{\omega}^{2}} \sin ^{2} \theta<1$. In the limit of $\theta \rightarrow \frac{\pi}{2}$, one finds $\tilde{R}_{\frac{\pi}{2}}^{4}=$ $\left(1+k^{2} / \tilde{\omega}^{2}\right) R_{\frac{\pi}{2}}^{4}$. The above equation is exactly the same form as in Eq.(355) with different " $R$ ". Thus the absorption cross section can be read off from (36) simply by substituting $R_{0}$ with $\tilde{R}_{\theta}$ 17

$$
\left.\sigma_{0}^{\varphi}\right|_{B}=\left.\sigma_{0}^{\varphi}\right|_{B=0}\left(R_{0} \rightarrow \tilde{R}_{\theta}\right)=\frac{\pi^{4}\left(\tilde{\omega} \tilde{R}_{\theta}\right)^{8}}{8 \omega^{5}}
$$

For an arbitrary $B$-field, one always finds $\left.\sigma_{0}^{\varphi}\right|_{B \neq 0}>\left.\sigma_{0}^{\varphi}\right|_{B=0}$ because of $\tilde{R}_{\theta}>R_{0}$.

In order to transform Eq. (34) into the familiar equation like Eq. (37), we redefine $\eta^{0}$ as $\eta^{0}=h^{-1 / 2} \hat{\eta}$. Then this leads to

$$
\left\{\frac{\partial^{2}}{\partial r^{2}}+\frac{5}{r} \frac{\partial}{\partial r}+\tilde{\omega}^{2}\left(1+\frac{\tilde{R}_{\theta}^{4}}{r^{4}}\right)+\frac{4 \sin ^{4} \theta R_{\theta}^{8} h^{2}}{r^{10} f^{4}}\right\} \hat{\eta}=0
$$

We can rewrite the last term in (39) in terms of $\tilde{R}_{\theta}, \tilde{f}=1+\tilde{R}_{\theta}^{4} / r^{4}, \tilde{h}^{-1}=\sin ^{2} \theta \tilde{f}^{-1}+\cos ^{2} \theta$ as

$$
\frac{\sin ^{4} \theta R_{\theta}^{8} h^{2}}{r^{10} f^{4}}=\frac{\sin ^{4} \theta \tilde{R}_{\theta}^{8} \tilde{h}^{2}}{r^{10} \tilde{f}^{4}}\left\{1-2 \epsilon\left(1-\frac{\tilde{R}_{\theta}^{4}}{r^{4}+\tilde{R}_{\theta}^{4}}-\frac{\tilde{R}_{\theta}^{4} \cos ^{2} \theta}{r^{4}+\tilde{R}_{\theta}^{4} \cos ^{2} \theta}\right)+\mathcal{O}\left(\epsilon^{2}\right)\right\}
$$

For the leading-order calculation, it is sufficient to keep the first term of the RHS of Eq.(40) only. Using $\hat{\eta}=r^{-5 / 2} \hat{\hat{\eta}}$, Eq. (39) leads to the Schrödinger-like equation as

$$
\left(\frac{\partial^{2}}{\partial r^{2}}+\tilde{\omega}^{2}-\tilde{V}_{\theta}\right) \hat{\hat{\eta}}=0
$$

where

$$
\tilde{V}_{\theta}=-\tilde{\omega}^{2}(\tilde{f}-1)+\frac{15}{4 r^{2}}-\frac{4 \sin ^{4} \theta R_{\theta}^{8} h^{2}}{r^{10} f^{4}}
$$


As will be shown in Eq.(65), the first term in Eq.(42) plays a role of energy term with $E=1$ in the near horizon of $r<R_{\theta}$. For $r>R_{\theta}$, the first term can be ignored. Thus we can approximate $\tilde{V}_{\theta}$ as $V_{\theta}$

$$
V_{\theta}=\frac{15}{4 r^{2}}-\frac{4 \sin ^{4} \theta R_{\theta}^{8} h^{2}}{r^{10} f^{4}}
$$

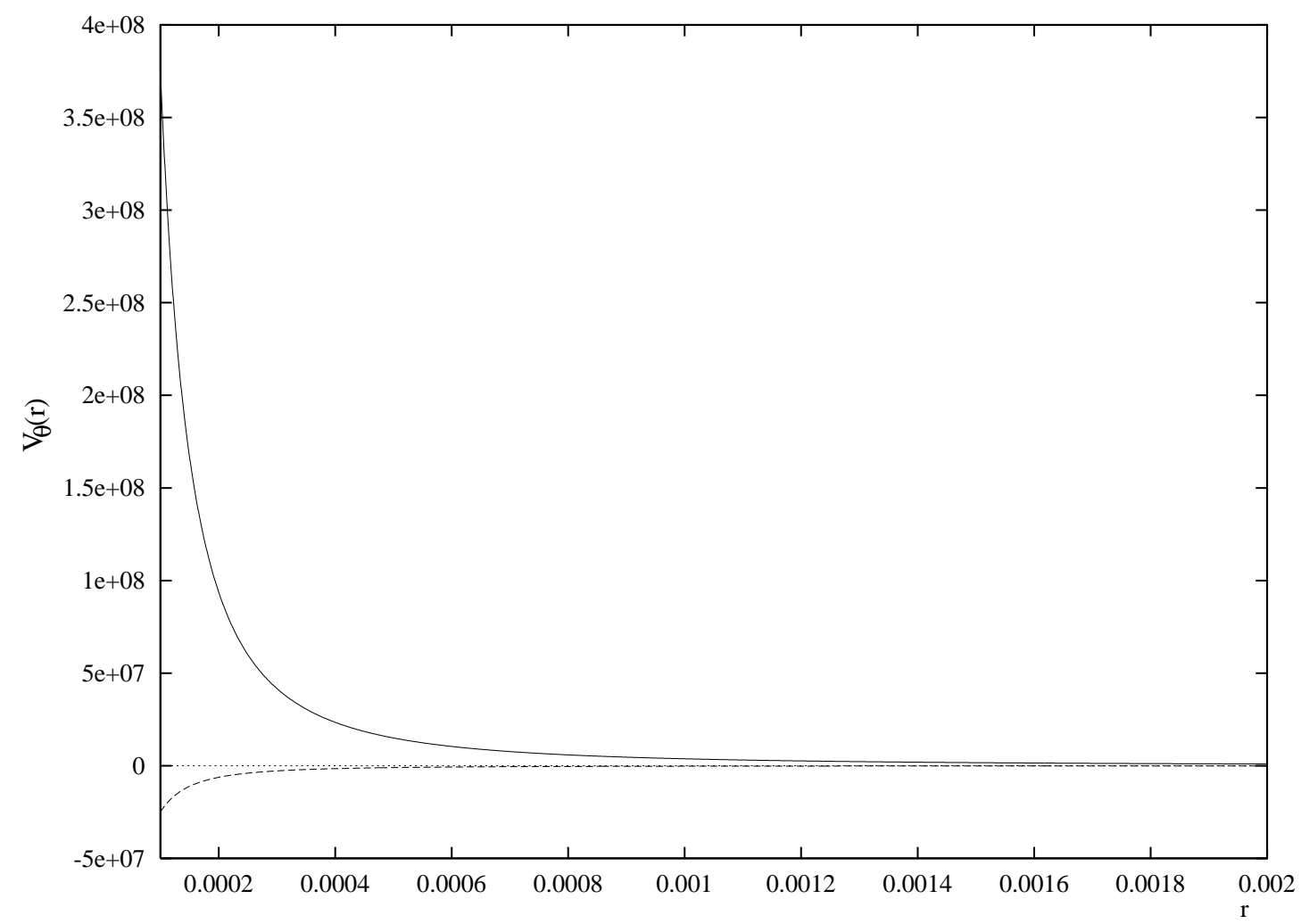

FIG. 1. The graphs of the RR scalar potential in the near horizon. For $\theta \rightarrow \pi / 2$, one finds a potential well(dashed line) and for $\theta=0$, one finds a potential barrier(solid line). The horizon is located at $r=0$.

For an arbitrary $\theta(B)$, it is very difficult to solve Eq. (41). Thus, let us discuss two interesting cases. If $\theta \simeq 0, h \simeq 1$. In this case, the last term of Eq.(43) can be neglected, compared with the first one. Then the RR scalar cross section takes the same form as that of the minimally coupled scalar in Eq. (37). For $\theta \rightarrow \frac{\pi}{2}, h \rightarrow f$. In this case, the last term of Eq.(43) plays an important role in the near-horizon. In the near horizon, one finds that $V_{\theta=0}^{N H}=15 / 4 r^{2}$ for $\theta=0$ and $V_{\theta \rightarrow \frac{\pi}{2}}^{N H}=-1 / 4 r^{2}$ for $\theta \rightarrow \pi / 2$. The latter case induces an instability of the nearhorizon geometry in the smeared D1-brane background because the potential well allows us 
the scattering $\operatorname{state}(\omega=$ real $)$ as well as the exponentially growing $\operatorname{state}(\omega=i \Omega)$. Also the same situation is recovered if one uses $\nabla^{2} \eta=0$ instead of Eq.(26). Hence the instability appears even for $\bar{H}_{M N P}=0$. As is shown in Fig.1, the singular behaviors of $V_{\theta=0, \frac{\pi}{2}}$ seem to appear as $r \rightarrow 0$. However, this is a coordinate artifact. Using the coordinate $z$ in Sec.VI, instead of $r$, one cannot find such singular behaviors in the near horizon.

\section{DILATON PROPAGATION WITH THE DILATON GAUGE}

In this section, we wish to study the propagation of the dilaton with the dilaton gauge in (31). Under this gauge the dilaton equation takes a rather simple form than the harmonic gauge [21]. Assuming

$$
\phi\left(t, x_{1}, x_{2}, x_{3}, r, \theta_{i}\right)=e^{-i \omega t} e^{i\left(k_{1} x_{1}+k_{2} x_{2}+k_{3} x_{3}\right)} Y_{l}\left(\theta_{1}, \theta_{2}, \cdots, \theta_{5}\right) \phi^{l}(r)
$$

the dilaton equation (27) leads to

$$
\begin{aligned}
\left\{\frac{\partial^{2}}{\partial r^{2}}\right. & +\frac{5}{r} \frac{\partial}{\partial r}-\frac{h^{\prime}}{h} \frac{\partial}{\partial r}-\frac{l(l+4)}{r^{2}}+\left(\omega^{2}-k_{1}^{2}\right) f-\frac{\left(k_{2}^{2}+k_{3}^{2}\right) f}{h} \\
& \left.+\frac{16 \sin ^{2} \theta \cos ^{2} \theta R_{\theta}^{8} h^{2}}{r^{10} f^{3}}+\frac{16 \sin ^{4} \theta R_{\theta}^{8} h^{2}}{r^{10} f^{4}}\right\} \phi^{l} \\
& +\frac{16 \sin ^{2} \theta \cos ^{2} \theta R_{\theta}^{8} h^{2}}{r^{10} f^{3}}\left\{f_{3}-\frac{1}{2}\left(h^{0}{ }_{0}+h^{1}{ }_{1}+h^{2}{ }_{2}+h^{3}{ }_{3}\right)\right\} \\
& +\frac{16 \sin ^{4} \theta R_{\theta}^{8} h^{2}}{r^{10} f^{4}}\left\{f_{3}-\frac{1}{2}\left(h_{0}^{0}+h_{1}^{1}+h_{r}^{r}\right)\right\}-\frac{10 \sin ^{2} \theta R_{\theta}^{4} h}{r^{6} f^{2}} h_{r}^{r}=0
\end{aligned}
$$

Our strategy is to disentangle the last three terms. For this purpose we have to use the dilaton gauge condition (31) and the linearized equations for $H, F_{3}, F_{5}$ in Eqs.(28)-(30). Because this is a nontrivial task for an arbitrary $\theta$, we only consider a simple and physically interesting case of $\theta \rightarrow \frac{\pi}{2}(B \rightarrow \infty)$. Here one choose the smeared D1-brane background with $\bar{H}_{M N P}=\bar{F}_{M N P Q R}=0$, which implies also that $h_{3}=f_{5}=0$. Then Eq.(28) with this gives us a constraint

$$
\left(\nabla_{M} \eta\right) \bar{F}^{M P Q}=0
$$


Since $\bar{F}^{M P Q} \neq 0$, Eq.(46) implies $\eta=0$. This means that $\eta$ is a non-propagating mode in the smeared D1-brane background. Hence the instability problem of the RR scalar arisen from the previous section is cured. The remaining ones are the s-wave dilaton equation and $F_{3}$-equation

$$
\begin{gathered}
\left\{\frac{\partial^{2}}{\partial r^{2}}+\frac{5}{r} \frac{\partial}{\partial r}+\left(\omega^{2}-k_{1}^{2}\right) f-\left(k_{2}^{2}+k_{3}^{2}\right)+\frac{4 R_{\frac{\pi}{2}}^{8}}{r^{10} f^{2}}\right\} \hat{\phi}-\frac{10 R_{\frac{\pi}{2}}^{8} h_{r}^{r}}{r^{6} f} \\
+\frac{16 R_{\frac{\pi}{2}}^{8}}{r^{10} f^{2}}\left\{\left(f_{3}-\frac{1}{2}\left(h_{0}^{0}+h^{1}{ }_{1}+h^{r}{ }_{r}\right)\right\}=0\right. \\
\nabla_{M} f^{M N P}-\left(\nabla_{M} h_{Q}^{N}\right) \bar{F}^{M Q P}+\left(\nabla_{M} h_{Q}^{P}\right) \bar{F}^{M Q N} \\
-\left(\nabla_{M} \hat{h}_{Q}^{M}\right) \bar{F}^{Q N P}-h_{Q}^{M}\left(\nabla_{M} \bar{F}^{Q N P}\right)=0 .
\end{gathered}
$$

Eq.(47) is derived from Eq. (45) with $\phi^{0}=h^{1 / 2} \hat{\phi}$ and $\theta=\frac{\pi}{2}$. In order to decouple the last term in (47), we have to use both Eq.(48) and the dilaton gauge Eq.(31). When $N=0, P=1$, solving Eq.(48) leads to [21]

$$
\partial_{r}\left(f_{3}-h_{0}^{0}-h_{1}^{1}\right)+\partial_{0} h_{r}^{0}+\partial_{1} h_{r}^{1}+\left(\frac{5}{r}+\frac{f^{\prime}}{f}\right) h_{r}^{r}=0 .
$$

Using the dilaton gauge, the last three terms turns out to be $\partial_{r}\left(-h^{r}{ }_{r}+\frac{1}{2} h\right)$. Then Eq.(49) gives us a crucial relation

$$
f_{3}-\frac{1}{2}\left(h_{0}^{0}+h_{1}^{1}+h_{r}^{r}\right)+\frac{1}{2}\left(h_{2}^{2}+h^{3}{ }_{3}+h_{\theta_{i}}^{\theta_{i}}\right)=0 .
$$

We point out that the same relation (50) can be found if one uses the harmonic gauge (32) 20. For simplicity, we can set $h_{\theta_{i}}^{\theta_{i}}=0$ and $h_{r}^{r}=0$. Then the equation (47) leads to

$$
\begin{gathered}
\left\{\frac{\partial^{2}}{\partial r^{2}}+\frac{5}{r} \frac{\partial}{\partial r}+\left(\omega^{2}-k_{1}^{2}\right) f-\left(k_{2}^{2}+k_{3}^{2}\right)+\frac{4 R_{\frac{\pi}{2}}^{8}}{r^{10} f^{2}}\right\} \hat{\phi} \\
-\frac{8 R_{\frac{\pi}{2}}^{8}}{r^{10} f^{2}}\left(h_{2}^{2}+h^{3}{ }_{3}\right)=0 .
\end{gathered}
$$

If the last term is absent, Eq. (51) reduces to the $\mathrm{RR}$ scalar equation (39) with $\theta=\frac{\pi}{2}$. It is easily proved that, considering (21) and (22) only, one finds that the dilaton equation (27) leads to Eq.(39). Hence the presence of the last term is important to distinguish the dilaton from the RR scalar. Without $B$-field, the fixed scalar $\lambda$ is given by [14] 


$$
\lambda=\frac{D-7}{2 \beta} \Phi-\frac{1}{2 \beta} \log V
$$

where $V$ is the world volume measured in $g_{M N}$. This implies that a trace of gravitons $\left(h^{a}{ }_{a}\right)$ polarized parallel in the world volume plays a role of the fixed scalar. With $B$-field, we may assume a relation between the dilaton and $h_{a}^{a}$. However, although we have a simple dilaton equation with the dilaton gauge, one cannot determine the relation between $\phi$ and $h_{2}^{2}+h^{3}{ }_{3}$. This is so because we have no further information for $h^{2}{ }_{2}+h^{3}{ }_{3}$. Hence we also have to use the Einstein equation( $(\mathrm{A} 2)$ in the smeared D1-brane background of Appendix $\mathrm{A}$ Using Eq.(A2) and (A3), one obtains a scalar equation

$$
R-4(\nabla \Phi)^{2}+4 \nabla^{2} \Phi=0
$$

For an s-wave propagation, it is sufficient to consider Eq.(53) instead of Eq.(A2). Its linearized equation takes the form

$$
\begin{gathered}
\bar{G}^{M N} \delta R_{M N}(h)-h^{M N} \bar{R}_{M N}-8(\nabla \bar{\Phi}) \cdot \nabla \phi-4 \nabla_{M} \bar{\Phi} \nabla_{N} \bar{\Phi} h^{M N} \\
-4 h^{M N} \nabla_{M} \nabla_{N} \bar{\Phi}-4 \bar{G}^{M N} \delta \Gamma_{M N}^{P} \nabla_{P} \bar{\Phi}+4 \nabla^{2} \phi=0
\end{gathered}
$$

with the Lichnerowitz operator [22]

$$
\begin{aligned}
\delta R_{M N}(h) & =-\frac{1}{2} \nabla^{2} h_{M N}-\frac{1}{2} \nabla_{M} \nabla_{N} h+\frac{1}{2} \nabla^{P} \nabla_{N} h_{P M}+\frac{1}{2} \nabla^{P} \nabla_{M} h_{P N} \\
& =-\frac{1}{2} \nabla^{2} h_{M N}-\bar{R}_{Q(M} h_{N)}^{Q}+\bar{R}_{P M Q N} h^{P Q}+\nabla_{(M} \nabla_{|P|} \hat{h}_{N)}^{P} .
\end{aligned}
$$

From Eq.(55) we obtain

$$
G^{M N} \delta R_{M N}=-\nabla^{2} h+\nabla_{P} \nabla_{N} h^{P N}
$$

The last term in (57) with the dilaton gauge gives rise to a difficult relation for $h_{M N}$ to solve Eq. (54). Hence we would be better to use the harmonic gauge condition (32) to obtain

$$
G^{M N} \delta R_{M N}=-\frac{1}{2} \nabla^{2} h
$$

which is also recovered from Eq.(56) with (32). 


\section{DILATON PROPAGATION WITH HARMONIC GAUGE}

The equation (54) leads to

$$
\begin{gathered}
\nabla^{2}\left(4 \phi-\frac{h}{2}\right)-\frac{4 f^{\prime}}{f^{3 / 2}} \phi^{\prime}-\frac{1}{f^{5 / 2}}\left(2 f f^{\prime \prime}-f^{\prime 2}\right) h_{r}^{r}-\frac{5 f^{\prime}}{2 r f^{3 / 2}} h_{\theta_{i}}^{\theta_{i}} \\
+\frac{f^{\prime 2}\left(h_{0}^{0}+h_{1}^{1}-h_{2}^{2}-h^{3}{ }_{3}+h^{r}{ }_{r}-h_{\theta_{i}}^{\theta_{i}}\right)}{2 f^{5 / 2}}=0 .
\end{gathered}
$$

Also the dilaton equation (27) takes the form

$$
\begin{aligned}
\nabla^{2} \phi-\frac{2 f^{\prime}}{f^{3 / 2}} \phi^{\prime}-\frac{f^{\prime \prime}}{2 f^{3 / 2}} h_{r}^{r}-\frac{2 f^{\prime}}{r f^{3 / 2}} h_{\theta_{i}}^{\theta_{i}} & \\
& +\frac{f^{\prime 2}\left\{h_{0}^{0}+h^{1}{ }_{1}-5\left(h^{2}{ }_{2}+h^{3}{ }_{3}+h^{\theta_{i}}{ }_{\theta_{i}}\right)\right\}}{8 f^{5 / 2}}=0
\end{aligned}
$$

Choosing Eq. (32) with $h=0$ (transverse-traceless gauge), one has $h_{0}^{0}+h^{1}{ }_{1}=-\left(h^{2}{ }_{2}+h^{3}{ }_{3}\right)$ with $h^{r}=h_{\theta_{i}}^{\theta_{i}}=0$. Then the above two equations become, respectively,

$$
\begin{gathered}
\left\{\frac{\partial^{2}}{\partial r^{2}}+\frac{5}{r} \frac{\partial}{\partial r}+\tilde{\omega}^{2}\left(1+\frac{\tilde{R}_{\frac{\pi}{2}}^{4}}{r^{4}}\right)\right\} \hat{\phi}-\frac{4 R_{\frac{\pi}{2}}^{8}\left(h^{2}{ }_{2}+h^{3}{ }_{3}\right)}{r^{10} f^{2}}=0 \\
\left\{\frac{\partial^{2}}{\partial r^{2}}+\frac{5}{r} \frac{\partial}{\partial r}+\tilde{\omega}^{2}\left(1+\frac{\tilde{R}_{\frac{\pi}{2}}^{4}}{r^{4}}\right)+\frac{4 R_{\frac{\pi}{2}}^{8}}{r^{10} f^{2}}\right\} \hat{\phi}-\frac{12 R_{\frac{\pi}{2}}^{8}\left(h^{2}{ }_{2}+h^{3}{ }_{3}\right)}{r^{10} f^{2}}=0 .
\end{gathered}
$$

The two equations (61) and (62) should be the same. Here we assume $h^{2}{ }_{2}+h^{3}{ }_{3}=a \phi$. Then one finds a relation

$$
4-12 a=-4 a
$$

which gives us $a=1 / 2$. Hence one obtains the correct dilaton equation as

$$
\left\{\frac{\partial^{2}}{\partial r^{2}}+\frac{5}{r} \frac{\partial}{\partial r}+\tilde{\omega}^{2}\left(1+\frac{\tilde{R}_{\frac{\pi}{2}}^{4}}{r^{4}}\right)-\frac{2 R_{\frac{\pi}{2}}^{8}}{r^{10} f^{2}}\right\} \hat{\phi}=0 .
$$

This can be approximated by using Eq. (40) as

$$
\left\{\frac{\partial^{2}}{\partial r^{2}}+\frac{5}{r} \frac{\partial}{\partial r}+\tilde{\omega}^{2}\left(1+\frac{\tilde{R}_{\frac{\pi}{2}}^{4}}{r^{4}}\right)-\frac{2 \tilde{R}_{\frac{\pi}{2}}^{8}}{r^{10} \tilde{f}^{2}}\right\} \hat{\phi} \simeq 0
$$


for the leading-order calculation. Finally, it remains to find an approximate solution to Eq.(65) for low energies $(\tilde{\omega} \rightarrow 0)$ and derive its absorption cross section. We divide the space into three regions $(I, I I, I I I)$ and then match solutions in them together. In the near horizon region $(I)$ the equation takes the form

$$
\left\{\frac{\partial^{2}}{\partial \rho^{2}}+\frac{5}{\rho} \frac{\partial}{\partial \rho}+\frac{\left(\tilde{\omega} \tilde{R}_{\frac{\pi}{2}}\right)^{4}}{\rho^{4}}-\frac{2}{\rho^{2}}\right\} \hat{\phi}_{I}(\rho)=0,
$$

where $\rho=\tilde{\omega} r$. Defining $\rho=\frac{\left(\tilde{\omega} \tilde{R}_{\frac{\pi}{2}}\right)^{2}}{z}$ and $\hat{\phi}_{I}(\rho)=z^{3 / 2} \hat{\hat{\phi}}_{I}$, this leads to

$$
\left\{\frac{\partial^{2}}{\partial z^{2}}+1-\frac{23}{4 z^{2}}\right\} \hat{\hat{\phi}}_{I}(z)=0
$$

which is nothing but the standard Bessel equation for $\hat{\hat{\phi}}_{I}(z)=H_{\sqrt{6}}(z)$. The above equation can be interpreted as the Schrödinger-like equation with the energy $E=1$ which is valid for large $z$ (in the near horizon of $r \rightarrow 0$ ). The solution is given by

$$
\hat{\phi}_{I}(z)=z^{2} H_{\sqrt{6}}(z)
$$

In the intermediate zone $(I I)$, the $\tilde{\omega}$-term can be ignored. Thus one finds the solution

$$
\hat{\phi}_{I I}(\rho)=C\left\{\frac{\rho^{4}}{\left(\tilde{\omega} \tilde{R}_{\frac{\pi}{2}}\right)^{4}+\rho^{4}}\right\}^{\frac{1}{2} \sqrt{\frac{3}{2}}-\frac{1}{2}} .
$$

In the far infinity region $(I I I)$ we have the equation

$$
\left(\frac{\partial^{2}}{\partial \rho^{2}}+\frac{5}{\rho} \frac{d}{d \rho}+\tilde{\omega}^{2}\right) \hat{\phi}_{I I I}(\rho)=0
$$

Its solution is given by

$$
\hat{\phi}_{I I I}(\rho)=D \frac{J_{2}(\rho)}{\rho^{2}}
$$

Matching $I I I$ to $I I$ leads to

$$
D=8 C
$$

Also matching $I$ to $I I$ gives 


$$
C=\frac{2^{\sqrt{6}}}{\pi} \Gamma(\sqrt{6})\left(\tilde{\omega} \tilde{R}_{\frac{\pi}{2}}\right)^{2-\sqrt{6}}
$$

Considering the ratio of the flux at the horizon $(r=0)$ to the incoming flux at infinity leads to the absorption probability as

$$
P_{\phi}=\frac{4}{|D|^{2}}\left(\tilde{\omega} \tilde{R}_{\frac{\pi}{2}}\right)^{8}=\frac{1}{16} \frac{\pi^{2}}{2^{2 \sqrt{6}}} \frac{\left(\tilde{\omega} \tilde{R}_{\frac{\pi}{2}}\right)^{2 \sqrt{6}+4}}{\Gamma(\sqrt{6})^{2}} .
$$

Finally, we obtain the s-wave absorption cross section of the dilaton in the limit of $B \rightarrow \infty$ as

$$
\begin{aligned}
\left.\sigma_{0}^{\phi}\right|_{B \rightarrow \infty} & =\frac{2^{5} \pi^{2}}{\omega^{5}} P_{\phi}=\frac{\pi^{4}}{2^{2 \sqrt{6}-1} \Gamma(\sqrt{6})^{2}} \frac{\left(\tilde{\omega} \tilde{R}_{\frac{\pi}{2}}\right)^{4+2 \sqrt{6}}}{\omega^{5}} \\
& \simeq \frac{\pi^{4}}{2^{2 \sqrt{6}-1} \Gamma(\sqrt{6})^{2}} \frac{\left(\tilde{\omega} \tilde{R}_{\frac{\pi}{2}}\right)^{8.9}}{\omega^{5}}
\end{aligned}
$$

\section{DISCUSSIONS}

First we discuss the propagation of fields in the smeared D1-brane background of $B \rightarrow$

$\infty$ limit. We have shown that, considering $h=h_{r}^{r}=h_{\theta_{i}}^{\theta_{i}}=0$, the dilaton $\phi, f_{3}$, and $h^{2}{ }_{2}+h^{3}{ }_{3}=-h^{1}{ }_{1}-h^{2}{ }_{2}=\phi / 2$ are physically propagating modes whereas the RR scalar $\eta$, $h_{3}$, and $f_{5}$, are non-propagating modes. Interestingly, it turns out that the absorption cross section of the dilaton in the limit of $B \rightarrow \infty$ is given by the replacement of $R_{0} \rightarrow \tilde{R}_{\frac{\pi}{2}}$ and $8 \rightarrow 8.9$ in Eqs. (36) and (75). The $R_{0} \rightarrow \tilde{R}_{\frac{\pi}{2}}(8 \rightarrow 8.9)$ arise from the presence of $B$-field(the couplings).

The RR scalar has a negative potential as shown in Fig.11. This induces an instability of the near horizon in the smeared D1-brane background. Fortunately, $H$-equation (28) requires that this mode should not be a propagating one.

For a general analysis, let us consider the following equation with the parameter $s$ upon the diagonalization:

$$
\left\{\frac{\partial^{2}}{\partial r^{2}}+\frac{5}{r} \frac{\partial}{\partial r}+\tilde{\omega}^{2}\left(1+\frac{\tilde{R}_{\frac{\pi}{2}}^{4}}{r^{4}}\right)-\frac{s \tilde{R}_{\frac{\pi}{2}}^{8}}{r^{10} \tilde{f}^{2}}\right\} \psi_{s}=0
$$


For $s>-4$, one obtains its absorption cross section as

$$
\left.\sigma_{0}^{\psi_{s}}\right|_{B \rightarrow \infty}=\frac{\pi^{4}}{2^{2 \sqrt{4+s}-1} \Gamma(\sqrt{4+s})^{2}} \frac{\left(\tilde{\omega} \tilde{R}_{\frac{\pi}{2}}\right)^{2 \sqrt{4+s}+4}}{\omega^{5}} .
$$

For the case of $s=32$ case, one finds an interesting cross section

$$
\left.\sigma_{0}^{\psi_{s=32}}\right|_{B \rightarrow \infty}=\frac{\pi^{4}}{2^{14} \cdot 15} \frac{\left(\tilde{\omega} \tilde{R}_{\frac{\pi}{2}}\right)^{16}}{\omega^{5}}
$$

which is the same order as in $h^{a}{ }_{a}$ in the absence of $B$-field and $k^{2}=0$ [14]

$$
\left.\sigma_{0}^{h^{a}{ }_{a}}\right|_{B=0}=\frac{\pi^{4}}{2^{17} \cdot 3^{4}} \frac{\left(\omega R_{0}\right)^{16}}{\omega^{5}}
$$

Hence we expect that the new scalars in the limit of $B \rightarrow \infty$ may take a value of $-4 \leq s \leq 32$. Especially, the RR scalar with $s=-4$ is not allowed for matching procedure and thus it cannot be a propagating mode. And the dilaton has $s=2$ and its absorption cross section is given by (75). For $s=0$ case(minimally coupled scalar), one can recover (38) with $\theta=\frac{\pi}{2}$ from (77).

In conclusion, the $B \rightarrow \infty\left(\theta \rightarrow \frac{\pi}{2}\right)$ limit is a delicate issue. Here for the calculation of the absorption cross section, we take only the limit of $\alpha^{\prime} \rightarrow 0$. In this case one finds $\bar{H} \propto \alpha^{\prime}, \bar{F}_{3} \propto 1 / \alpha^{\prime}, \bar{F}_{5} \propto$ finite. It is known that $\bar{F}_{5}$ counts the rank of the noncommutative group. However, $\bar{F}_{5} \neq 0$ and $\bar{H} \neq 0$ leads to the complicated coupled equations. Solving these coupled equations is a formidable task. We remind the reader that the fluxes of $\bar{F}_{5}$ and $\bar{H}$ can be neglected in comparison with that of $\bar{F}_{3}$. Hence we choose the simple smeared D1-brane background by setting $\bar{F}_{5}=\bar{H}=0$. At the first sight, this action seems to be eliminating any connection to noncommutative geometry. However, although we do not count the fluxes of $F_{5}$ and $H$ thoroughly, we still give the effect of the noncommutativity on the absorption cross section through $\tilde{R}_{\frac{\pi}{2}}^{4} \gg R_{0}^{4}$ in $f=1+\tilde{R}_{\frac{\pi}{2}}^{4} / r^{4}$ and the important coupling of $F_{3}$. If $\bar{H} \neq 0$ and $\bar{F}_{5} \neq 0$, we expect that there will be a change in $s: R_{0} \rightarrow \tilde{R}_{\frac{\pi}{2}}$, $8 \rightarrow s(2 \leq s<32)$. This is so because the coupling scheme will change " $s$ ".

Finally we comment the ways to account the noncommutative effect on the cross section of the dilaton on the supergravity side. These are $R_{\frac{\pi}{2}}^{4} \gg R_{0}^{4}, k^{2} \neq 0$, and the couplings to 
all other fields. Here we include the expansion of the parameter $R_{\frac{\pi}{2}}^{4}\left(\gg R_{0}^{4}\right)$, the presence of momenta along the world volume directions $\left(k_{2}, k_{3}\right)$ to detect $B_{23}$-field, and the coupling of $F_{3}$ with $H=F_{5}=0$. Analysis for an arbitrary $\theta(B)$ remains unexplored.

\section{ACKNOWLEDGEMENT}

Authors would like to thank Sangmin Lee for helpful discussion. This work was supported by the Brain Korea 21 Program, Ministry of Education, Project No. D-0025.

\section{APPENDIX A: THE SMEARED D1-BRANE SOLUTION}

In the case of $F_{5}=H_{3}=\chi$, the string frame action takes the form

$$
S_{10}^{\mathrm{SD} 1}=\frac{1}{2 \kappa_{10}^{2}} \int d^{10} x \sqrt{-G}\left[e^{-2 \Phi}\left\{R+4(\nabla \Phi)^{2}-\frac{1}{12} F_{3}^{2}\right\}\right]
$$

which leads to the equations of motion

$$
\begin{aligned}
& R_{M N}=-2 \nabla_{M} \nabla_{N} \Phi+\frac{1}{4} e^{2 \Phi} F_{M P Q} F_{N}^{P Q}-\frac{1}{24} e^{2 \Phi} F_{3}^{2} G_{M N} \\
& \nabla^{2} \Phi-2(\nabla \Phi)^{2}-\frac{1}{12} e^{2 \Phi} F^{2}=0 \\
& \nabla_{M} F_{3}^{M P Q}=0 .
\end{aligned}
$$

The smeared D1-brane solution is given by

$$
\begin{aligned}
& d s_{\mathrm{SD} 1}^{2}=f^{-\frac{1}{2}}\left\{-d x_{0}^{2}+d x_{1}^{2}+f\left(d x_{2}^{2}+d x_{3}^{2}\right)\right\}+f^{\frac{1}{2}}\left(d r^{2}+r^{2} d \Omega_{5}^{2}\right), \\
& f=1+\frac{C}{r^{4}}, \quad e^{2 \bar{\Phi}}=g^{2} f, \quad \bar{F}_{01 r}=\frac{1}{g} \partial_{r}\left(f^{-1}\right) .
\end{aligned}
$$

Here "C" is an arbitrary constant, but in order to make connection to the noncommutative geometry we have to choose $C=R_{\frac{\pi}{2}}^{4}=4 \pi \tilde{g} \tilde{b} \tilde{N} \gg R_{0}^{4}$. 


\section{REFERENCES}

[1] A. Connes and M. Douglas, JHEP 9802, 003(1998), hep-th/9711162.

[2] M. Douglas and C. Hull, JHEP 9802, 008(1998), hep-th/9711165.

[3] M.M. Sheikh-Jabbari, Phys. Lett. B450, 119(1999), hep-th/9810179.

[4] A. Hashimoto and N. Itzhaki, hep-th/9907166.

[5] J. Maldacena and J. Russo, hep-th/9908134.

[6] N. Seiberg and E. Witten, hep-th/9908142.

[7] D. Bigatti and L. Susskind, hep-th/9908056.

[8] J. Maldacena, Adv. Theor. Math. Phys. 2, 231(1998), hep-th/9711200.

[9] A. Hashimoto and N. Itzhaki, hep-th/9911057.

[10] Y.S. Myung and H.W. Lee, hep-th/9911031; hep-th/9910083.

[11] R.G. Cai and N. Ohta, hep-th/9910092.

[12] S. Gubser, I. Klebanov, and A. Tseytlin, Nucl. Phys. B499, 217(1997), hep-th/9703040; S. Gubser and I. Klebanov, Phys. Lett. B413, 41(1997), hep-th/9708005; S. Gubser, I. Klebanov, and A. Polyakov, Phys. Lett. B428, 105(1998), hep-th/9802109.

[13] S. Gubser and A. Hashimoto, Comm. Math. Phys. 203, 325(1999); hep-th/9805140.

[14] S. Gubser, A. Hashimoto, I. Klebanov, and M. Krasnitz, Nucl. Phys. B526, 393(1998), hep-th/9803023.

[15] H.W. Lee and Y.S. Myung, Phys. Rev. D58, 104013(1998), hep-th/9804095; hepth/9903054; H.W. Lee, N.J. Kim, and Y.S. Myung, hep-th/9805050.

[16] S. Hyun, Y. Kiem, S. Lee, and C.Y. Lee, hep-th/9909059.

[17] Y.S. Myung, G. Kang, H.W. Lee, hep-th/9911193. 
[18] A. Kaya, hep-th/9911183.

[19] S.R. Das, S.K. Rama, and S.P. Trivedi, hep-th/9911137.

[20] H.W. Lee, Y.S. Myung, and J.Y. Kim, JHEP 9910, 014(1999), hep-th/9907024; Phys. Lett. B466, 211(1999), hep-th/9908092.

[21] H.W. Lee, N.J. Kim, Y.S. Myung, and J.Y. Kim, Phys. Rev. D57, 7361(1998), hepth/9801152.

[22] R. Gregorgy and R. Laflamme, Phys. Rev. Lett. 70, 2837(1993); Nucl. Phys. B428, 399(1994), hep-th/9404071. 